\title{
Solar cycle as a dissipative magnetic structure
}

\author{
A.A. Soloviev and E.A. Kirichek \\ Central (Pulkovo) astronomical observatory, St-Petersburg; solov@gao.spb.ru
}

\begin{abstract}
The dissipative model of Solar Magnetic Cycle (SMC) is discussed. According to this conception, at the beginning of each odd 11-yr cycle, a large-scale dissipative magnetic structure is formed in the convective zone of the Sun, as a result of complex nonlinear interaction between magnetic field and turbulent plasma velocity field. During the 22-yr cycle, this large-scale magnetic structure evolves through the convective "envelope" and atmosphere of the Sun. Two main effects determine the process: i) diffusion of magnetic field and ii) $\omega$-effect: the conversion of poloidal magnetic field into toroidal one due to the differential rotation.
\end{abstract}

Keywords. diffusion, magnetic fields, MHD

\section{The diffusion model of SMC and "arrow of time"}

The diffusion-relaxation model of SMC was, basically, formulated by Soloviev \& Kirichek (2004). The main idea is that the observable 22-year magnetic cycle (the Hale's cycle) is formed by a certain indivisible large-scaled dissipative structure of magnetic field. It is generated in the convective zone. After, it dissipates through the Sun interior and looses its energy due to the turbulent diffusion. The process takes 22 years. Then the initial magnetic structure goes away irreversibly to the interplanetary space. The process starts again: new magnetic structure, similar but independent on the previous one, emerges into convective zone from its bottom and stipulates all complexity of SMC. The principal prediction of our model was: the magnetic structure, that forms entire SMC, should irreversibly vanish, decaying and "getting older" due to the process of energy dissipation. "Arrow of time" on 22-yr timescale results in a following effect: any characteristics of dissipative processes, related to the entropy of magnetic system, should change gradually during the 22 -yr cycle and return abruptly to the initial state at the origin of new cycle, when the old structure goes away. In fact, this effect was discovered by Nagovitsyn (1988) as early as 1988. He studied the variations of correlation between different adjacent "energetical" and "temporal" characteristics of 11-year cycles inside the 22-year SMC to generalize the known Gnevychev-Ohl rule. The traditional Rule relates even 11-year cycle with the following odd one: "energetical" characteristics (the areas under cyclic curve) reveal maximal correlation in even+odd couples. However, the "temporal" characteristics (times between neighboring extremes) reveal strong correlation, with coefficient 0.94 , within odd+even couple (e. g. $21^{\text {st }}$ and $22^{\text {nd }}$ cycles). This correlation, being maximal in the beginning of the odd cycle, decays monotonically and vanishes during the following 22 years. This phenomenon can be explicitly explained as manifestation of "Arrow of Time", i.e. as increasing of entropy (decreasing of the order of the system) during the SMC. The entropy peaks when the old structure is on the verge to escape the Sun, then decreases abruptly when the new magnetic structure emerges. The "Arrow of Time" acts on $22 \mathrm{yr}$ timescale (not on the $11 \mathrm{yr}$ one). All this contradicts the Dynamo models, which traditionally assumes regeneration of poloidal field $B_{P}$ every 11 yr due to the "alpha effect". 

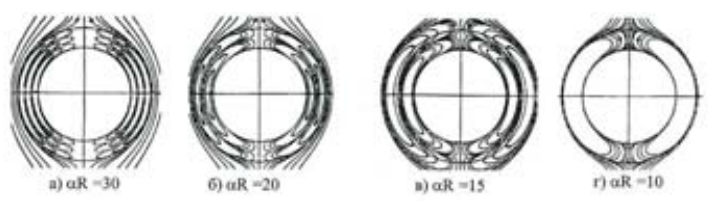

Figure 1. Example of diffusion evolution.

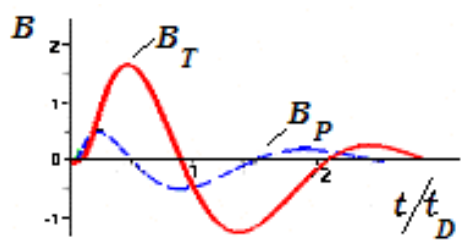

Figure 2. Action of diffusion and omega-effect.

\section{Possible geometry and time evolution of dissipative structure}

Now we can't understand how the large-scale magnetic dissipative structure is formed in the convective zone of the Sun, being the result of complex nonlinear interaction between the turbulence and magnetic field; but we can derive some conclusions about its geometry in its resulting state. We base on two general assumptions: i) quasi-stationary condition: the work produced by magnetic field over the turbulent plasma (in time interval greater then mechanical relaxation time of the system, but much less then $22 \mathrm{yr}$ ) is to be equal to the work produced by the turbulent motions over magnetic field, ii) the axial symmetry. It can be shown, that the first condition is satisfied if the magnetic force has the potential (gradient) of form $[\operatorname{rot} \mathbf{B} \times \mathbf{B}]=4 \pi \nabla \tilde{P}$, where $\tilde{P}(r, t)$ is the perturbation of gas pressure distribution caused by magnetic field. We introduce the magnetic flux, in spherical coordinates $(r, \theta, \varphi)$, by relation $\Psi=-\int_{0}^{t} B_{\theta} \sin \theta r d r$ and assume $B_{\varphi}=$ $\frac{\alpha_{0} R}{r \sin \theta} \Psi$, where $\alpha_{0}$ is a constant. In case of pure diffusion problem (i.e. $[\mathbf{V} \times \mathbf{B}]=\nabla \chi$ ), we can derive the fundamental solution for the magnetic flux $\Psi$ :

$$
\Psi(\tilde{r}, \theta, \tilde{t})=B_{0} r_{0}^{2} \sin ^{2} \theta \sqrt{\tilde{r}} \sum_{k} \sum_{i} C_{i} \exp \left(-\alpha_{i}^{2} R^{2}\left(\tilde{t}-\tilde{t}_{0 i}\right) J_{k+1 / 2}\left(\alpha_{i} R \tilde{r}\right) P_{\mathrm{k}}^{\prime}(\cos \theta) .\right.
$$

Here $P_{k}^{\prime} \cos \theta$ is the derivative of Legendre polynomial of degree $k$ with respect to $\cos \theta$; $k=0,1,2,3 \ldots, B_{0}, C_{i}, \alpha_{i}, t_{0, i}$ are constants. The variables are dimensionless: $\tilde{r}=r / r_{0}$, $\tilde{t}=t / t_{D}\left(r_{0}\right)$, where $r_{0}$ is the magnetic field scale, and $t_{D}\left(r_{0}\right)=r_{0}^{2} \nu_{m}^{-1}$ is the skin (diffusion) time for the $r_{0}$. Every harmonic term of solution (2.1) gives a set of axially symmetric toroids, inserted into the spherical layer. Any initial field distribution can be represented as the superposition of terms in the form of series (2.1) with the different scale parameters $\alpha_{i}$. Diffusion will first eliminate the terms with greater $\alpha_{i}$ and then the terms with small $\alpha_{i}$. Such disproportionate decrease of harmonics will result in the diffusion spreading of the field, i.e. in propagation of the field outwards, to the outer space. To present the process in more simple and clear form we can regard the time-evolution of one harmonics with variable scale-parameter $\alpha_{i}$. The Fig. 1 shows the evolution of three magnetic toroids $(k=1)$ embedded, at the initial moment, into the convective zone of the Sun. The picture of magnetic toroids spreading due to diffusion outwards can explain a number of features of SMC development; in particularly, the reversals of global field occur when the boundary between the adjacent magnetic toroids passes through the surface.

The diffusion solution (2.1) can be generalized for the case $\operatorname{rot}[\mathbf{v} \times \mathbf{B}] \neq 0$, i.e. when the omega-effect takes place. In that case, the quasi-periodic time variations of basic harmonics can be derived as for the toroidal, $B_{T}$, and poloidal, $B_{P}$, field, on the interval about 22 yr. (Fig. 2).

\section{References}

Soloviev A.A., Kirichek E.A. The diffusion theory of Solar Magnetic Cycle. Elista-SanctPeterburg, Kalmyk University-GAO RAS, 2004. - 181 P

Nagovitsyn Yu. A. Solnechnye dannye, 1988, 12, P. 109 